Mots. Les langages du politique

Trente ans d'étude des langages du politique

(1980-2010)

\title{
Discours politiques, propagande, communication, manipulation
}

\section{Caroline Ollivier-Yaniv}

\section{OpenEdition}

\section{Journals}

Édition électronique

URL : https://journals.openedition.org/mots/19857

DOI : $10.4000 /$ mots. 19857

ISSN : 1960-6001

Éditeur

ENS Éditions

\section{Édition imprimée}

Date de publication : 30 novembre 2010

Pagination : $31-37$

ISBN : 978-2-84788-235-3

ISSN : 0243-6450

\section{Référence électronique}

Caroline Ollivier-Yaniv « Discours politiques, propagande, communication, manipulation » Mots. Les langages du politique [En ligne], 94 | 2010, mis en ligne le 06 novembre 2012, consulté le 28 avril 2022. URL : http://journals.openedition.org/mots/19857 ; DOI : https://doi.org/10.4000/mots. 19857 


\section{Mots \\ Les langages du politique}

№ 94 novembre 2010

\section{Trente ans d'étude des langages du politique (1980-2010)}

ouvrage coordonné par Paul BACOT, Marlène Coulomb-GulLY, Jean-Paul Honoré, Christian Le BART, Claire Oger, Christian Plantin

\section{SOMMAIRE}

Paul Bacot, Marlène Coulomb-Gully, Jean-Paul Honoré, Christian Le Bart, Claire Oger, Christian Plantin

Le discours politique n'est pas transparent. Permanence

et transformations d'un objet de recherche

OUTILS ET ENJEUX DU DISCOURS POLITIQUE

Ruth Amossy, Roselyne Koren

Argumentation et discours politique

Christian Plantin

Argumentation-rhétorique. Les eaux mêlées

Caroline Ollivier-Yaniv

Discours politiques, propagande, communication, manipulation 
Marc Bonhomme

La caricature politique

Paul Bacot

Développement et diversification d'une onomastique politique

Ruth Wodak

The Discursive Construction of History. Brief Considerations

Henri Boyer

Les politiques linguistiques

\section{LIEUX DE LA PRODUCTION DU DISCOURS POLITIQUE}

Christian Le Bart

Parler en politique

Dominique Maingueneau

Le discours politique et son «environnement»

Alice Krieg-Planque, Claire Oger

Discours institutionnels. Perspectives pour les sciences

de la communication

Sophie Béroud, Josette Lefèvre

Le corpus syndical. Une expérience au long cours

Corinne Gobin, Jean-Claude Deroubaix

L'analyse du discours des organisations internationales.

Un vaste champ encore peu exploré

François de la Bretèque

Le retour de la parole politique dans le cinéma français

Jean-Claude Soulages

Vie et mort du citoyen cathodique

Jacques Guilhaumou

Les discours de la Révolution française.

Aperçu d'ensemble d'un trajet de recherche (1980-2009) 


\section{DISCIPLINES ET CHAMPS DE RECHERCHE POUR L'ÉTUDE DES LANGAGES DU POLITIQUE}

Philippe Braud

L'apport de la science politique à l'étude des langages du politique

Claire Blandin

L'apport de l'histoire des médias à l'étude des langages du politique

Jean-François Tétu, Bernard Lamizet

Les SIC et les langages du politique

Sylvianne Rémi-Giraud

Sémantique lexicale et langages du politique.

Le paradoxe d'un mariage difficile?

Marlène Coulomb-Gully, Juliette Rennes

Genre, politique et analyse du discours.

Une tradition épistémologique française gender blind

Johannes Angermüller

Analyser le discours politique en Allemagne (1980-2010)

Érik Neveu

L'apport de Pierre Bourdieu à l'analyse du discours.

D'un cadre théorique à des recherches empiriques

Roselyne Ringoot

Questionner le discours avec Michel Foucault.

Actualisations théoriques et actualité éditoriale

\section{ENTRETIEN}

Maurice Tournier

Mots et politique, avant et autour de 1980

La revue Mots. Les langages du politique encourage l'usage des rectifications de l'orthographe proposées par le Conseil supérieur de la langue française et approuvées par l’Académie (Journal officiel, $n^{\circ}$ 100, 6 décembre 1990). 


\section{Caroline Ollivier-Yaniv}

\section{Discours politiques, propagande, communication, manipulation}

En démocratie, la propagande, la communication politique et la manipulation sont des notions qui servent à caractériser la circulation des discours politiques entre les professionnels de la politique et les citoyens, au moyen des médias de masse et avec un objectif de persuasion ou d'imposition de sens. Ces termes présentent toutefois une particularité : leurs usages dans la littérature scientifique ne sont pas équivalents, un peu à la manière de ce que l'on peut observer dans le discours des responsables politiques ou des journalistes. Les trente dernières années constituent une période particulièrement pertinente pour observer ce phénomène. En effet, la tension entre les catégories «propagande» et «communication politique» est devenue particulièrement manifeste dans les années quatre-vingt, décennie caractérisée par la construction de la communication comme catégorie d'analyse dominante, dans la sphère politique et au-delà. Cette tension se cristallise autour de la notion de manipulation, c'està-dire de la potentialité d'influencer et de transformer (to spin) les idées ou les comportements des citoyens sans qu'ils en aient conscience, en s'adressant à leurs émotions plutôt qu'à leur raison ou encore en faisant usage du mensonge ou de la désinformation. Autrement dit, la référence à la manipulation, péjorative depuis l'invention du sujet politique au $18^{\mathrm{e}}$ siècle (D'Almeida, 2005), conduit à disqualifier les pratiques et les discours politiques.

Les études qui portent sur propagande, communication et manipulation depuis trente ans, en France, peuvent être analysées à partir de leur positionnement relativement à cette tension entre propagande et communication : trois perspectives sont ainsi distinguées dans ce texte. On rend compte tout d'abord des travaux qui explicitent le remplacement de la propagande par la communication politique; ensuite, des travaux qui s'attachent à mettre en évidence que la manipulation des discours politiques n'a pas disparu de l'espace public démocratique et qu'elle lui est même consubstantielle; enfin, des travaux qui s'attachent plutôt à analyser les contraintes de fabrication, de circulation et de réception des discours politiques dans une société de « relations publiques généralisées» (Miège, 1997). 


\section{La propagande remplacée par la communication politique}

On peut identifier deux grands ensembles d'études qui signifient le remplacement de la propagande par la communication politique. Les premiers s'inscrivent dans la perspective théorique de l'espace public habermassien et définissent la communication politique comme condition de fonctionnement d'un espace public élargi. Les seconds s'inscrivent dans une perspective historique et s'attachent à définir les caractéristiques de la communication politique contemporaine en les différenciant de ses ancêtres propagandistes.

La définition de la communication politique proposée par Dominique Wolton en 1989, dans l'un des premiers numéros de la revue Hermès, est celle d'un «espace où s'échangent les discours des trois acteurs qui ont la légitimité à s'exprimer publiquement sur la politique et qui sont les hommes politiques, les journalistes et l'opinion publique au travers des sondages » (Wolton, 1989, p. 39). Le premier objectif de cette définition est de réhabiliter la part discursive et symbolique de la politique, c'est-à-dire d'un ensemble d'activités qui ont souffert de leur assimilation à la propagande dans le cadre des régimes politiques totalitaires. Le second objectif consiste à mettre en évidence que la communication politique est synonyme du bon fonctionnement d'un espace public élargi et par suite, d'une extension de la démocratie. Ici, la communication politique apparaît comme une construction théorique démocratique idéale puisque la possibilité de la confrontation de ces trois discours, dont l'auteur admet qu'ils peuvent se trouver en tension, est le signe de la reconnaissance de l'autre. Cette définition dialogique, presque délibérative, de la communication politique exclut la possibilité de la manipulation et de la propagande - ou les relègue au rang de "dérives» (Dacheux, 2004, p. 30) - parce qu'elle oblige l'émetteur à prendre en considération tous les autres acteurs démocratiquement légitimes.

On retrouve cette définition de la communication politique comme «idéal démocratique de dialogue et d'échange» (Delporte, 2007, p. 459) dans des travaux qui émanent de l'histoire des médias. La différenciation entre communication politique et propagande est étayée par deux critères principaux. Le premier est le pluralisme des médias et la liberté de circulation de l'information. De ce point de vue, la propagande devient caractéristique des régimes totalitaires ou de situations exceptionnelles dans les régimes démocratiques, par exemple en cas de conflit (Bertho-Lavenir, 2000). Le second critère est le degré de prise en considération, par l'émetteur politique, de l'état de l'opinion publique : «La communication politique, c'est d'abord la recherche d'un dialogue avec l'opinion publique, d'un échange permettant d'établir avec elle un contrat fondé sur la confiance. C'est pourquoi les sondages (dont neuf sur dix restent aujourd'hui confidentiels) sont capitaux» (Delporte, 2007, p.10). L'affirmation du «bascu- 
lement» d'un «âge de la propagande à un âge de la communication » voisine toutefois régulièrement avec la reconnaissance de ses «ambivalences» (Delporte, 2003, p.3-4) : l'approche historique signifie ainsi la nécessité de dissocier plus nettement l'idéal démocratique de ses conditions d'actualisation.

Cette différenciation d'avec la propagande (en tant que celle-ci est unidirectionnelle et non dialogique) et, par suite, la disparition de celle-ci comme catégorie démocratique sous-tendent la plupart des ouvrages qui s'attachent à décrire les procédés et les enjeux de la communication politique en général (Gerstlé, 2008).

\section{La dénonciation de la communication politique comme forme renouvelée de la manipulation}

D'autres travaux s'attachent au contraire à mettre en évidence, éventuellement sur le mode de la révélation, voire de la dénonciation, que la communication politique constitue une forme renouvelée de la manipulation des discours et des symboles dans le cadre démocratique, celle-ci lui étant consubstantielle. La distinction entre propagande et communication n'a alors plus lieu d'être, les deux catégories renvoyant à une communauté de pratiques et surtout d'objectifs.

La thèse selon laquelle la manipulation et la propagande n'ont de pertinence que dans le cadre démocratique a été particulièrement développée par Philippe Breton. Le caractère libéral du régime démocratique, son utilisation par définition limitée de la coercition mais encore la dimension concurrentielle de la sphère publique rendent nécessaire la mise au point de «méthodes spécifiques de conquête du consensus » (Breton, 1997, p.72) et de techniques de manipulation de la parole. De manière apparemment paradoxale au regard du sens commun, la manipulation des discours est définie comme étant plus caractéristique des régimes démocratiques que des régimes totalitaires. Sur un plan plus strictement linguistique, la manipulation est distinguée de l'argumentation en ce qu'elle pratique l'amalgame ou, pour reprendre l'expression d'Aristote, «le parler hors de la cause» (Breton, 2003).

D'un point de vue politique, cette réflexion n'est pas sans lien avec la définition de la propagande d'État proposée par le politiste Jacques Ellul dès les années cinquante (Ellul, 1952, 1962) : celle-ci n'a de raison d'être que dans une sphère publique pluraliste, au sein de laquelle d'autres organisations (économiques en particulier) font usage de stratégies de persuasion de l'opinion publique.

La communication politique apparaît ainsi comme une forme renouvelée - révélée par les auteurs - de la manipulation des discours politiques dans un régime démocratique. Pour caractériser ses modes opératoires, Philippe Breton parle de «violence indirecte, cachée, hypocrite, qui est essentiellement 
une violence séductrice»(Breton, 2009, p.40). À partir d'une toute autre approche, le sociologue Jacques Le Bohec qualifie de «tour de passe-passe linguistique » la «transfiguration méliorative de la propagande en "communication" »(Le Bohec, 1997, p.52).

La révélation des mécanismes et des procédés manipulatoires - avec leurs objectifs explicites d'influence - sous-jacents au développement de la communication politique contemporaine a fait l'objet de plusieurs ouvrages récents. Il en est ainsi de la mise au jour du storytelling comme procédé de construction de récits (à savoir une succession de séquences mettant en scène un héros) en vue d'obtenir des effets sur l'opinion (Salmon, 2007), ou encore de la republication (en 2007) de l'ouvrage d'Edward Bernays (1928), l'un des premiers professionnels des relations publiques et de la manipulation (du spin) aux ÉtatsUnis. À côté d'une utile contextualisation du texte, la préface de cette nouvelle édition est l'occasion de développer une démonstration par la preuve - la manipulation existe en démocratie puisque l'un de ses spécialistes et de ses fondateurs la dévoile - ainsi que de désapprouver la dénaturation cynique de l’idéal démocratique dont elle est synonyme.

\section{La propagande comme interdit et la communication politique comme obligation. Analyser les discours politiques sous contrainte}

On peut enfin identifier un troisième ensemble de travaux qui interrogent de manière plus générale la dimension discursive du métier politique ou de l'action publique, ou encore qui prennent plus particulièrement en considération les transformations des contraintes politiques, institutionnelles, médiatiques et économiques qui pèsent sur la fabrication, la diffusion et la circulation des discours politiques dans le cadre des démocraties représentatives (Quaderni, 2010).

L'analyse du discours étudie ainsi les transformations du discours politique du fait de sa médiatisation, considérant notamment l'existence de «savoir-faire relatifs à l'anticipation des pratiques, de transformation et de reformulation des énoncés et de leurs contenus » (Krieg-Planque, 2006, p. 34). Discours politiques et médiatiques semblent alors constituer un ensemble hybride, caractérisé par l'émergence d'énoncés diversifiés (slogans, "petites phrases ») aux propriétés stylistiques et rhétoriques ainsi qu'aux modes de circulation spécifiques (Rinn, 2002).

Du point de vue de la sociologie politique (Riutort, 2007), l'analyse du développement des ingénieries symboliques et de leurs professionnels dans la sphère politique fait l'objet de nombreux travaux qui explorent l'émergence, l'institutionnalisation ainsi que les croyances d'acteurs sociaux spécialistes du travail symbolique auprès des responsables politiques (Legavre, 1993; Georga- 
kakis, 1995) et dans les institutions (Ollivier-Yaniv, 2000 ; Nollet, 2006), mais encore les interdépendances entre professionnels de la politique, spécialistes de la communication et journalistes (Ollivier-Yaniv, 2001; Thomas, 2007) ou les mécanismes de coproduction de l'information à partir des interactions entre les journalistes et leurs sources institutionnelles (Bastin, 2002).

Lorsqu'elles sont explicitement abordées, les catégories de "propagande » - objet de rejet, de déni (Georgakakis, 2004) ou de disqualification - ou de " communication » - en tant qu'elle est au contraire devenue un impératif (Oger, 2003; Ollivier-Yaniv, 2010) - sont constituées non plus en tant que cadres d'analyse mais bien en tant qu'objets dont il convient d'analyser et de contextualiser les usages discursifs et sociaux.

La référence à la manipulation est considérée avec circonspection; elle peut même se trouver réfutée pour des raisons axiologiques (Soulez, 2004). Sa mobilisation comme catégorie d'analyse présente en effet le risque de présupposer une intention cynique ou une représentation hyperstratégique des acteurs et des métiers politiques, au risque - scientifique - de négliger la complexité des facteurs explicatifs des rapports qu'entretiennent les " professionnels du travail symbolique à leur activité » (Neveu, 1994, p. 116). Elle peut également laisser à penser que l'analyste méconnaît ou occulte le fait que les effets des stratégies de persuasion sont caractérisés par une forte incertitude (Le Bart, 2001, p. 47) et qu'il cède à la croyance dans l'efficacité de la manipulation et dans l'existence d'une intention manipulatoire.

On le voit, la mise en perspective de trente années d'études sur discours politiques, propagande, communication et manipulation conduit à revenir sur la mobilité des frontières existant entre ces catégories. À certains égards, les travaux dits scientifiques ou académiques paraissent traversés par des enjeux normatifs, autrement dit par un clivage entre ce qui est conforme aux idéaux démocratiques (la liberté d'expression et de circulation de tous les discours politiques) et ce qui ne l'est pas (la manipulation), à la manière d'autres discours qui leur sont contemporains et qui peuvent être qualifiés d'indigènes parce qu'ils sont portés par des acteurs sociaux impliqués dans la mise en œuvre de ces phénomènes (responsables politiques, journalistes, communiquants). Autrement dit, la tension entre propagande, communication et manipulation apparaît révélatrice de l'hiatus entre le présupposé d'un idéal démocratique et ses conditions de réalisation, mais encore d'une confusion entre la démocratie comme modèle, d'une part, et comme forme politique complexe, d'autre part.

Partant, la caractérisation de propagande, communication et manipulation en tant que "catégories historiographiques» (Olivesi, 2002, p.13) se trouve ainsi confortée. Leur construction se trouverait sans doute encore éclairée par des analyses sociohistoriques de leurs usages, à la fois dans les discours dits 
savants et dans les discours des «montreurs de communication » (Neveu, 1994, p.104). Il y a donc encore beaucoup à dire de la propagande, de la communication, de la manipulation et de leurs relations du point de vue de la sociohistoire des discours et des pratiques politiques.

\section{Références}

BASTIN G., 2002, «Les journalistes accrédités auprès des institutions européennes à Bruxelles. Quelques signes du changement d'un monde de travail», Les métiers de l'Europe politique. Acteurs et professionnalisations de l'Union européenne, D. Georgakakis éd., Strasbourg, Presses universitaires de Strasbourg, p. 169-194.

Bernays E., 2007 [1928], Propaganda. Comment manipuler l'opinion en démocratie, préface N. Baillargeon, Paris, La Découverte.

Bertho-LAVENIR C., 2000, La démocratie et les médias au xxe siècle, Paris, Armand Colin. BRETON P., 1997, La parole manipulée, Paris, La Découverte.

- 2003, "Le "plaider en dehors de la cause" d'Aristote. Un critère technique pour séparer argumentation et manipulation?», Argumentation et discours politique, S. Bonnafous, P. Chiron, D. Ducard, C. Lévy éd., Rennes, Presses universitaires de Rennes, p. 153-163.

- 2009, "La démocratie, matrice de la propagande? ", Communication de l'État et gouvernement du social, C. Ollivier-Yaniv, M. Rinn éd., Grenoble, Presses universitaires de Grenoble, p. 39-47.

D’AlmEIDA F., 2005, La manipulation, Paris, PUF.

DACHEUX E., 2004, L'impossible défi. La politique de communication de l'Union européenne, Paris, CNRS.

DELPORTE C., 2007, La France dans les yeux. Une histoire de la communication politique de 1930 à nos jours, Paris, Flammarion.

- 2003, "Pour une histoire de la propagande et de la communication politique», Vingtième siècle. Revue d'histoire, $n^{\circ} 80$, p. 3-4.

ElLuL J., 1952, «Propagande et démocratie», Revue française de sciences politiques, $\mathrm{n}^{\circ} 3, \mathrm{p} .473-504$.

- 1962, Propagandes, Paris, Economica.

GEORGAKAKIS D., 1995, "La double figure des conseils en communication politique », Sociétés contemporaines, $\mathrm{n}^{\circ}$ 24, p.77-94.

- 2004, La République contre la propagande. Aux origines perdues de la communication d'État en France (1917-1940), Paris, Economica.

GeRSTLÉ J., 2008, La communication politique, Paris, Armand Colin.

KrIEg-Planque A., 2006, “Formules” et “lieux discursifs”. Propositions pour l'analyse du discours politique», entretien par P. Schepens, Semen. Revue de sémiolinguistique des textes et discours, $\mathrm{n}^{0} 21, \mathrm{p} .19-47$.

LE BART C., 2001, Le discours politique, Paris, PUF.

LE BOHEC J., 1997, Les rapports presse-politique, Paris, L'Harmattan. 
LEGAVRE J.-B., 1993, "Conseiller en communication politique. L'institutionnalisation d'un rôle», thèse de doctorat en science politique, Université Paris 1.

MıÈGE B., 1997, La société conquise par la communication. La communication entre l'industrie et l'espace public, t. II, Grenoble, Presses universitaires de Grenoble.

NeVEu É., 1994, Une société de communication?, Paris, Montchrestien.

NOLLET J., 2006, "Les communicateurs de ministère entre champ bureaucratique et journalistique», Journalisme et dépendance, I. Chupin, J. Nollet éd., Paris, L’Harmattan, p. 161-185.

OGER C., 2003, "Communication et contrôle de la parole. De la clôture à la mise en scène de l'institution militaire», Quaderni, n²52, Secret et pouvoir. Les faux-semblants de la transparence, p. 77-92.

OLIVESI S., 2002, «De la propagande à la communication. Éléments pour une généalogie», Cahiers d'histoire. Revue d'histoire critique, n86, Démocratie, pouvoirs et propagande en France au xxe siècle, p. 13-28.

OLLIVIER-YANIV C., 2000, L'État communiquant, Paris, PUF.

- 2001, "L'indépendance des journalistes à l'épreuve du politique et de la communication. Le localier, le chargé de communication publique et l'élu local», Quaderni, $n^{\circ} 45$, Figures du journalisme. Critique d'un imaginaire professionnel, p. 87-104.

- 2010, "De l'opposition entre "propagande" et "communication publique" à la définition de la politique du discours. Proposition d'une catégorie analytique», Quaderni, $n^{\circ} 72$, Propagandes en démocratie, p. 87-100.

SALMON C., 2007, Storytelling. La machine à fabriquer des histoires et à formater les esprits, Paris, La Découverte.

Soulez G., 2004, "Rhétorique, public et “manipulation” », Hermès, n³8, p. 89-95.

Quaderni, 2010, nº72, Propagandes en démocratie, E. Taïeb éd.

RINN M., 2002, Les discours sociaux contre le sida. Rhétorique de la communication publique, Bruxelles, De Boeck Université.

RIUTORT P., 2007, Sociologie de la communication politique, Paris, La Découverte.

ThomAS C., 2007, «Journalistes cherchent bons communiquants. L'exemple des rubricards justice», Les publics des journalistes, P. Dauvin, J.-B. Legavre éd., Paris, La Dispute, p. 19-43.

Wolton D., 1989, «La communication politique. Construction d'un modèle », Hermès, $\mathrm{n}^{\circ} 4$, Le nouvel espace public, p. 27-42. 